\title{
Optical and Electrical Properties of Te Doped AlGaAsSb/AlAsSb Bragg Mirrors on InP
}

\author{
D. O. Toginho Filho, I. F. L. Dias, J. L. Duarte, E. Laureto, \\ Departamento de Física, Universidade Estadual de Londrina, CP 6001, CEP 86051-970, Londrina-Paraná, Brazil \\ and Jean C. Harmand \\ CNRS, Laboratoire de Photonique et de Nanostructures, Route de Nozay, 91460 Marcoussis, France
}

\author{
Received on 22 August, 2006
}

\begin{abstract}
We present a comparative study carried out on the optical and electrical characteristics of undoped and Te doped AlGaAsSb/AlAsSb Bragg mirrors with 6.5 pairs of layers and bulk undoped and Te doped AlGaAsSb epilayers alloys lattice matched on InP, grown by molecular beam epitaxy, using SIMS, photoluminescence, reflectivity and $\mathrm{IxV}$ techniques. The temperature dependence of PL transitions observed in the Bragg mirrors are similar to that observed in bulk samples and associated with the donor and acceptor recombinations in alloys with electrostatic potential fluctuations described by quasi-donor-acceptor-pair (QDAP) models. We verified by SIMS the presence of a macro fluctuation in the Te profile concentrations in the growth direction of the doped Bragg mirror. The influence of doping, alloy and macrofluctuation of incorporated Te on the optical and electrical properties of doped Bragg mirror is analyzed. The AlGaAs/AlAsSb seems a very attractive option for VCSEL technology.
\end{abstract}

Keywords: Semiconductor; Bragg mirror; AlGaAs/AlAsSb

\section{INTRODUCTION}

Semiconductor materials of the (Al)GaAsSb family lattice matched on InP have been used for heterojunctions structures employed in optoelectronic devices in the range of 1.0 $\mu \mathrm{m}$ e $2.0 \mu \mathrm{m}$ [1-3]. Due to their high refractions index contrast systems, the $\mathrm{Sb}$ materials such as AlGaAsSb/AlAsSb [4] and $\mathrm{GaAsSb} / \mathrm{AlAsSb}[5]$ are considered good alternatives for the preparation of distributed Bragg mirrors (DBR) [6], a key component in optoelectronic devices as vertical-cavity surface emitting lasers (VCSEL).

In ternary and quaternary alloys employed in the preparation of DBRs , the phase separation resulting from miscibility gaps induces compositional inhomogeneities in the crystal $[7,8]$. The average potential depth increases with the number of components in the alloy and can act on optical and electrical properties of devices by creating scattering centers to transport charge carriers and/or band tails in the band gap. It is shown, for example, that the presence of $\mathrm{Al}$ in the quaternary $\mathrm{AlGaAsSb}$ alloy increases the fluctuation of the electrostatic potential in the epitaxial layer as compared to the observed in the ternary GaAsSb [9].

The required properties for DBRs are a reflectivity exceeding $99 \%$ and a low electrical serial resistance. High reflectivity is accomplished by employing materials with a high refractive contrast $(\Delta n)$ and a sufficient number of quarter wave layers. Low electrical resistance requires a sufficiently high doping level and a low potential discontinuity $\Delta \mathrm{Ec}$ in the band conduction ( $\Delta E v$ in the band valence) for n-type (p-type) [10] mirrors. However, systems with high $\Delta \mathrm{n}$ also present a high band discontinuity $(\Delta \mathrm{Eg})$ which is not favorable to the current flow through the heterointerfaces. Raising the doping level will lower the serial resistance at the expense of an increase in free carrier absorption and a series of changes in the physical properties of the semiconductor materials as a reduction in impurity ionization energy [10-12], a shift in the funda- mental absorption edge [13-15], an increase in the density of the states in the vicinity of the band limits and a reduction in the fundamental gap $[16,17]$. Then a compromise must be found to achieve the high reflectivity and the low resistance at the same time. High quality undoped quarter wave stacks transparent to $1.55 \mu \mathrm{m}$ wavelength were realized using $\mathrm{Al}_{x} \mathrm{Ga}_{1-x} \mathrm{As}_{y} \mathrm{Sb}_{1-y}$ lattice matched to InP with $\mathrm{x}$ around 0.10 $[18,19]$

The quaternary alloy $\mathrm{Al}_{x} \mathrm{Ga}_{1-x} \mathrm{As}_{y} \mathrm{Sb}_{1-y}$ lattice matched on InP presents a direct $\Gamma$ valley for $0 \leq \mathrm{x} \leq 0.40$. For $\mathrm{AlAs}_{y} \mathrm{Sb}_{1-y}$ lattice matched on InP the lowest conduction band valley is the indirect $\mathrm{X}$ for any $\mathrm{y}$ value. The conduction band off-set $\Delta \mathrm{E}_{C}(\Gamma-\mathrm{X})$ in the $\mathrm{AlGaAsSb} / \mathrm{AlAsSb}$ system is approximately $\cong 470 \mathrm{meV}$ in the typical $\mathrm{Al}$ concentration for Bragg mirror operating in the $1.55 \mu \mathrm{m}$ range $(\mathrm{x} \cong 0.1)$. A relatively high doping level and/or some metalurgical intervention at the interfaces in the structure are needed to improve electrical conduction.

Due to the low $\mathrm{Al}$ concentration required to prepare the 1.55 $\mu \mathrm{m}$ Bragg mirror with $\mathrm{AlGaAsSb} / \mathrm{AlAsSb}$, the properties of the quaternary alloy are similar to those of the ternary alloys GaAsSb. Nominally undoped GaAsSb shows non-excitonic residual acceptor impurity related to native defects as gallium vacancies and gallium on antimony sites $\left(\mathrm{V}_{G a} \mathrm{Ga} S b\right)$ with concentrations of $10^{16} \mathrm{~cm}^{-3}$. PL measurements on undoped $\mathrm{GaAsSb} / \mathrm{InP}$ and AlGaAsSb/InP show a dominant transition identified as DAP (donor-acceptor-pair) associated with the electrostatic potential fluctuations in agreement with QDAP model. QDAP transitions are also identified in PL measurements of Te doped AlGaAsSb grown by molecular beam epitaxy lattice matched to InP. In doped and compensated materials, the random distribution of donor and acceptor impurities also causes a fluctuation of the electrostatic potential in the semiconductors layers. The conduction and valence bands are distorted by the random potential , and the behavior of PL transitions can be explained by the QDAP model. 
The potential fluctuations associated with the compositional inhomogeneity and/or donor and acceptor impurities can interfere in the transport an optical properties of the alloys employed in the preparation of DBRs. Therefore the study of the electrical and optical properties of undoped and Te doped $\mathrm{AlGaAsSb} / \mathrm{AlAsSb}$ Bragg mirrors compared to those of undoped and doped mirror component epilayers of $\mathrm{AlGaAsSb}$ is important for the development of VCELs technology.

Here we report a systematic experimental work to make comparisons between the optical electrical and optical properties of bulk undoped and Te doped layers of AlGaAsSb and undoped and Te doped AlGaAsSb/AlAsSb Bragg mirrors employing Secondary Ion Mass Spectometry - SIMS, photoluminescence (PL), reflectivity and IxV measurements.

\section{EXPERIMENTAL DETAILS}

Undoped and Te doped AlGaAsSb/AlAsSb Bragg mirrors with 6.5 pairs were grown by MBE lattice matched on a (100) InP:Fe and (100) InP-n substrates, respectively. Te doped bulk AlGaAsSb and AlAsSb samples lattice matched on a (100) InP:Fe were also grown by MBE. The carrier concentrations in the Te doped samples $\left(\cong 10^{18} . \mathrm{cm}^{-3}\right)$ were determined by Hall measurements, at room temperature, using Van der Paw methods. Details of the sample parameters such as alloy composition, doping levels and structural parameters of Bragg mirrors can be seen in Table I.

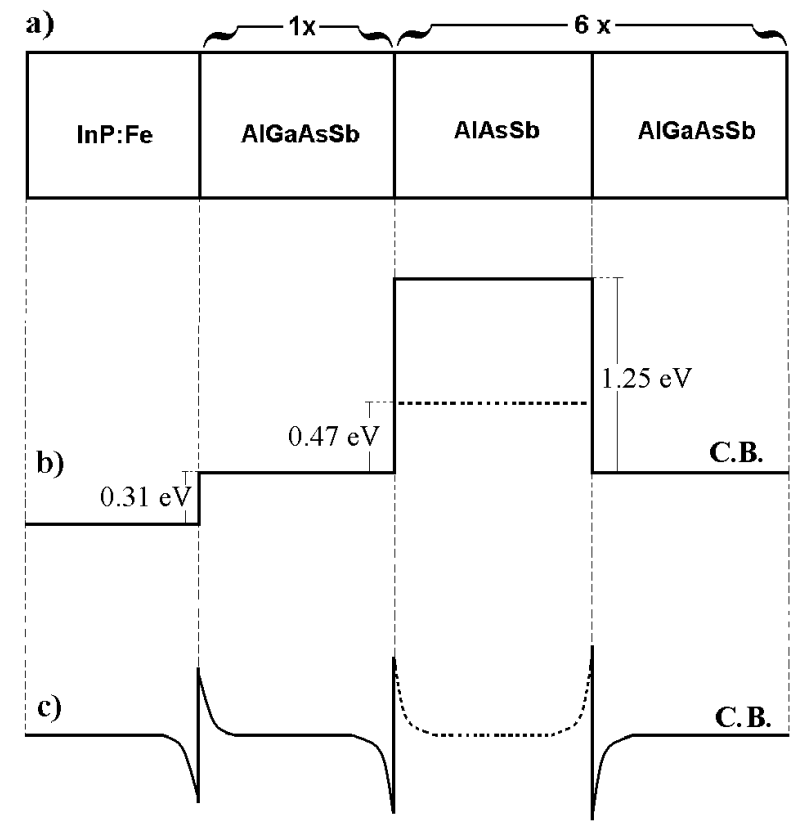

FIG. 1: - a) Structure diagram of the Bragg mirrors; b) Potential profile diagram of the conduction band in undoped Bragg mirror; c) Potential profile diagram of the conduction band in doped Bragg mirror.

The structure of Bragg mirrors with 6.5 pairs and the conduction band diagram for one period of undoped and Te doped
AlGaAsSb/AlAsSb samples are shown in Fig. 1a), 1b) and 1c), respectively. The band conduction alignment with the offset between $\mathrm{Al}_{0.1} \mathrm{Ga}_{0.9} \mathrm{As}_{0.52} \mathrm{Sb}_{0.48}, \mathrm{AlAs}_{0.56} \mathrm{Sb}_{0.44}$ (X valley in dotted lines) and the substrate InP are also shown in Fig. 1b). The concentration profile of alloy constituents and the Te doping were obtained using SIMS.

PL measurements were performed in the temperature range of $10 \mathrm{~K}$ to $300 \mathrm{~K}$, using the $514.5 \mathrm{~nm}$ line of a continuous wave $\mathrm{Ar}^{+}$laser, with different excitation intensities. Temperature variation was obtained by a closed-cycle helium cryostat. The spectral analyses of the luminescence measurements were carried out by a $0.5 \mathrm{~m}$ Jarrel-Ash spectrometer, coupled to a cooled InGaAs photodetector, using the standard lock-in technique.

Experimental reflectivity was measured with a Fourier transform infrared spectrometer (FTIR) in the so-called VW configuration. This configuration provided an absolute measurement of the reflectivity and the real shape of the reflectivity spectrum with quantitative values at a $0.2 \%$ level of accuracy.

The n-type doped Bragg mirror was metallized with Ti/Au ohmic contacts evaporated on the top and on the bottom of the sample. The sample was patterned with $100 \times 100 \mu \mathrm{m}^{2}$ square mesas etched by an $\mathrm{H}_{2} \mathrm{SO}_{4}: \mathrm{H}_{2} \mathrm{O}_{2}: \mathrm{H}_{2} \mathrm{O}$ solution. The substrate underside was thinned to $150 \mu \mathrm{m}$ to decrease the substrate contribution to the electrical measurements. The current-voltage $(\mathrm{IxV})$ dc characteristic is made by the transmission line method (TLM) measurement.

\section{RESULTS AND DISCUSSION}

The SIMS profile of Te concentrations in bulk Te doped $\mathrm{AlAsSb}$ and AlGaAsSb (dotted lines) alloys prepared with similar growth conditions as that analyzed in this work is shown in Fig. 2a). The two samples show a uniform pattern for the doping level of tellurium with the depth in the growth direction. The SIMS profile of the constituent materials (Al, $\mathrm{Ga}, \mathrm{As}, \mathrm{Sb}, \mathrm{Te}$ ) of the Te doped AlGaAsSb and AlAsSb layers of Bragg mirror of 6.5 periods are presented in Fig. 2b). The incorporated Te shows a periodic fluctuation in the growth direction which is not observed in the bulk samples as shown in Fig. 2a). This unexpected fluctuation in the n-type dopant concentration can be associated with the growth process. The temperature of tellurium Knudsen cell in the growth chamber $\left(\cong 185^{\circ} \mathrm{C}\right)$ is very low when compared to the characteristic temperatures of Knudsen cells for other materials as Al ( $\cong 1100^{\circ} \mathrm{C}$ ). The on/off control of shutters located in front of the $\mathrm{Al}$ cells, should interfere in the temperature conditions of the Te cell. The automatic system normally found in growth chambers to control the stability of the temperatures should change the temperature of the Te cell and therefore of the material flux for a time interval. However, this is a speculation that needs experimental corroboration. There is no evidence that Te activity (and probably the Te incorporation) depends on the Al mole fraction [18]. The same pattern in the SIMS profile is observed in other Te doped Bragg mirror samples with 6.5 pairs layers grown in the same or other growth series. 
TABLE I: Stoichiometric composition, thickness and Te concentration of the $\mathrm{Al}_{x} \mathrm{Ga}_{1-x} \mathrm{As}_{y} \mathrm{Sb}_{1-y}$ bulk samples and $\mathrm{AlGaAsSb} / \mathrm{AlAsSb} \mathrm{Bragg}$ mirror with 6,5 períods.

\begin{tabular}{|c|c|c|c|c|c|c|}
\hline \multirow[t]{2}{*}{ Bulk samples } & \multicolumn{3}{|c|}{ Nominal thickness( nm) } & \multicolumn{2}{|c|}{$\begin{array}{l}\text { composition } \\
\mathrm{Al}_{x} \mathrm{Ga}_{1-x} \mathrm{As}_{y} \mathrm{Sb}_{1-y}\end{array}$} & \multirow[t]{2}{*}{ Carrier concentration $\left(\mathrm{cm}^{-3}\right)$} \\
\hline & & & & $\mathrm{x}$ & $\mathrm{y}$ & \\
\hline $76 \mathrm{~N} 46$ & \multicolumn{3}{|l|}{$\cong 600$} & 0,08 & 0,51 & undoped \\
\hline $76 \mathrm{~N} 39$ & \multicolumn{3}{|l|}{$\cong 600$} & 0,08 & 0,51 & $2,4 \times 10^{18}$ \\
\hline \multirow{2}{*}{ Mirror samples } & \multicolumn{3}{|c|}{ Nominal thickness( $\mathrm{nm})$} & & & \multirow{2}{*}{$\mathrm{AlGaAsSb} / \mathrm{AlAsSb}$} \\
\hline & $\mathrm{AlGaAsSb}$ & $\mathrm{AlAsSb}$ & total & & & \\
\hline 17Q29 & 111,5 & 130,8 & 1565 & 0,10 & 0,51 & undoped \\
\hline 17Q44 & 108,3 & 127,1 & 1521 & 0,10 & 0,51 & $3,0 / 1,8$ \\
\hline
\end{tabular}

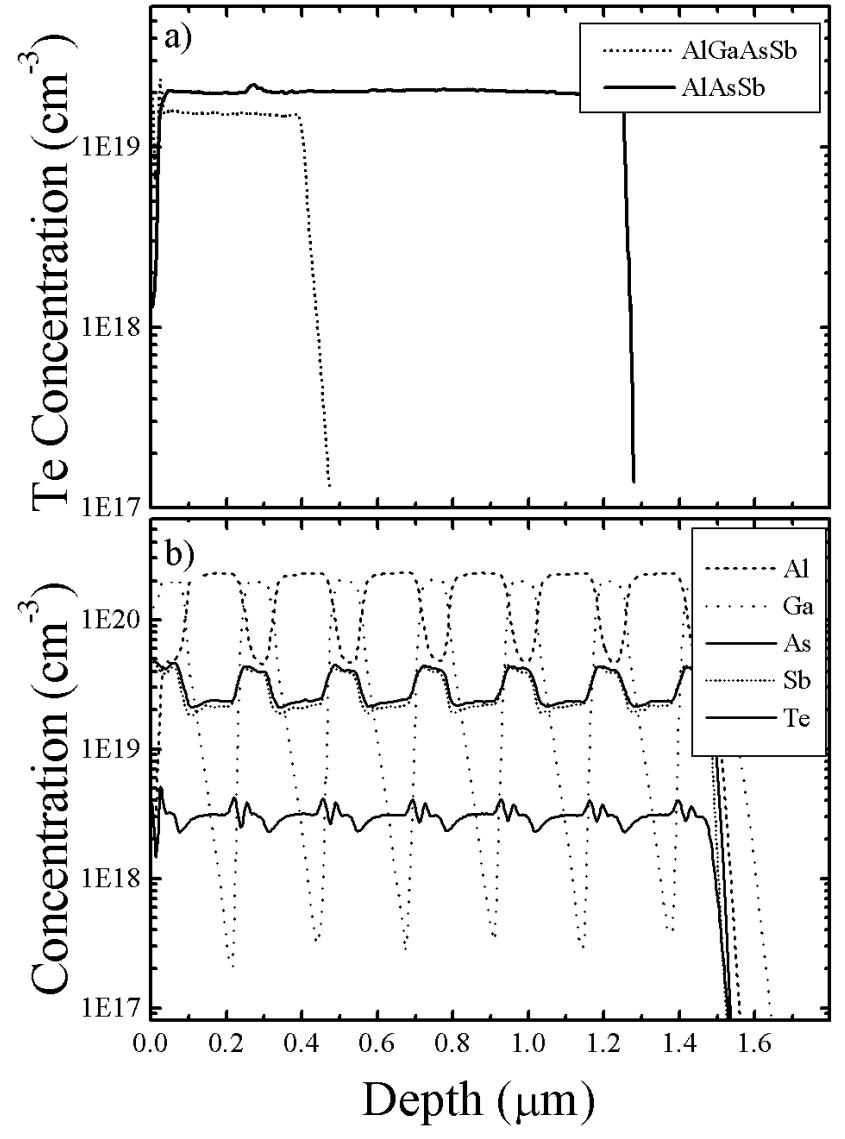

FIG. 2: a) Te concentration profile in AlAsSb and AlGaAsSb doped bulk samples, growth in similar conditions at the doped bulk samples analised in this work; b) Concentration profile of the elements that compose th doped homogeneous Bragg mirror 17Q44.

Figure 3 shows the PL spectra at $10 \mathrm{~K}$ and $300 \mathrm{~K}$ of undoped bulk AlGaAsSb alloy (76N46) and n-type Te doped bulk Al$\mathrm{GaAsSb}(76 \mathrm{~N} 39)$ with carrier concentration of $2.4 \times 10^{18} \cdot \mathrm{cm}^{-3}$ in comparison with PL spectra of undoped Bragg mirror (17Q29) and Te doped Bragg mirror (17Q44) with a carrier concentration of $3.0 \times 10^{18} . \mathrm{cm}^{-3}$. The analyzed spectra in the Bragg mirror are related to the AlGaAsSb layers because the ternary AlAsSb alloy is pratically transparent in the $1.55 \mu \mathrm{m}$ region. The PL spectra of undoped bulk sample (Fig. 3a) and undoped Bragg mirror (Fig. 3b) show very similar lineshape at $10 \mathrm{~K}$ and $300 \mathrm{~K}$. The energy shift between the PL transitions and the broadening of emission spectrum in the Bragg mirror as compared to that obtained in the bulk alloy is a result of the higher $\mathrm{Al}$ concentration in the Bragg mirror (see Table I).

The PL spectra of Te doped bulk sample (Fig. 3c) and Te doped Bragg mirror (Fig. 3d)) show similar results. The PL principal peak of Bragg mirror is shifted a few meV to higher energy and show higher broadening as compared to that obtained for the bulk sample. This is a consequence of higher $\mathrm{Al}$ concentration and higher doping level in the Bragg mirror epilayers $\left(8 \% \mathrm{Al}\right.$ and $2,4 \times 10^{18} \cdot \mathrm{cm}^{-3}$ in the bulk versus $10 \%$ $\mathrm{Al}$ and $3,0 \times 10^{18} . \mathrm{cm}^{-3}$ in the mirror).

The PL spectra of undoped Bragg mirror at $10 \mathrm{~K}$ with excitation intensity variation in the range $0.57 \mathrm{~W} / \mathrm{cm}^{2}$ to 532 $\mathrm{W} / \mathrm{cm}^{2}$ in linear and $\log$ scale are presented in Fig. 4a) and Fig. 4b), respectively. The dominant PL transition shifts to a higher energy region with increasing excitation intensity. In PL spectra obtained at low temperature, the increase in the excitation intensity rises the number of photogenerated free carriers that screens the electrostatic potential, decreasing the amplitude of potential fluctuations and, consequently, increasing the energy of the emission peak. The spectra in log scale show a secondary peak at $\cong 33 \mathrm{meV}$ associated with a LOphonon replica [20] below the principal transition visible for excitation intensities higher than $10.6 \mathrm{~W} / \mathrm{cm}^{2}$. A set of PL spectra with excitation intensity fixed at $532 \mathrm{~W} / \mathrm{cm}^{2}$ recorded at increasing temperature for the undoped Bragg mirror is shown in linear scale in the Fig. 4c) and in the log scale in Fig. 4d). The dominant PL peak energies show a characteristic inverted " $\mathrm{S}$ " behavior as reported elsewhere for bulk samples. The secondary peak at $\cong 33 \mathrm{meV}$ of the principal transition can be also seen in the log scale spectra still at $50 \mathrm{~K}$.

The PL spectra of Te doped Bragg mirror as a function of intensity and temperature can be seen in Fig. 5. In Figs. 5a) (linear scale) and 5b) (log scale) we have the spectra obtained at $10 \mathrm{~K}$ with laser excitation intensity between $1.06 \mathrm{~W} / \mathrm{cm}^{2}$ and $532 \mathrm{~W} / \mathrm{cm}^{2}$. The PL peak is very broad, suggesting the presence of another transition in the lower energy region. This transition is more evident at $5.7 \mathrm{~W} / \mathrm{cm}^{2}$. The PL spectra at 


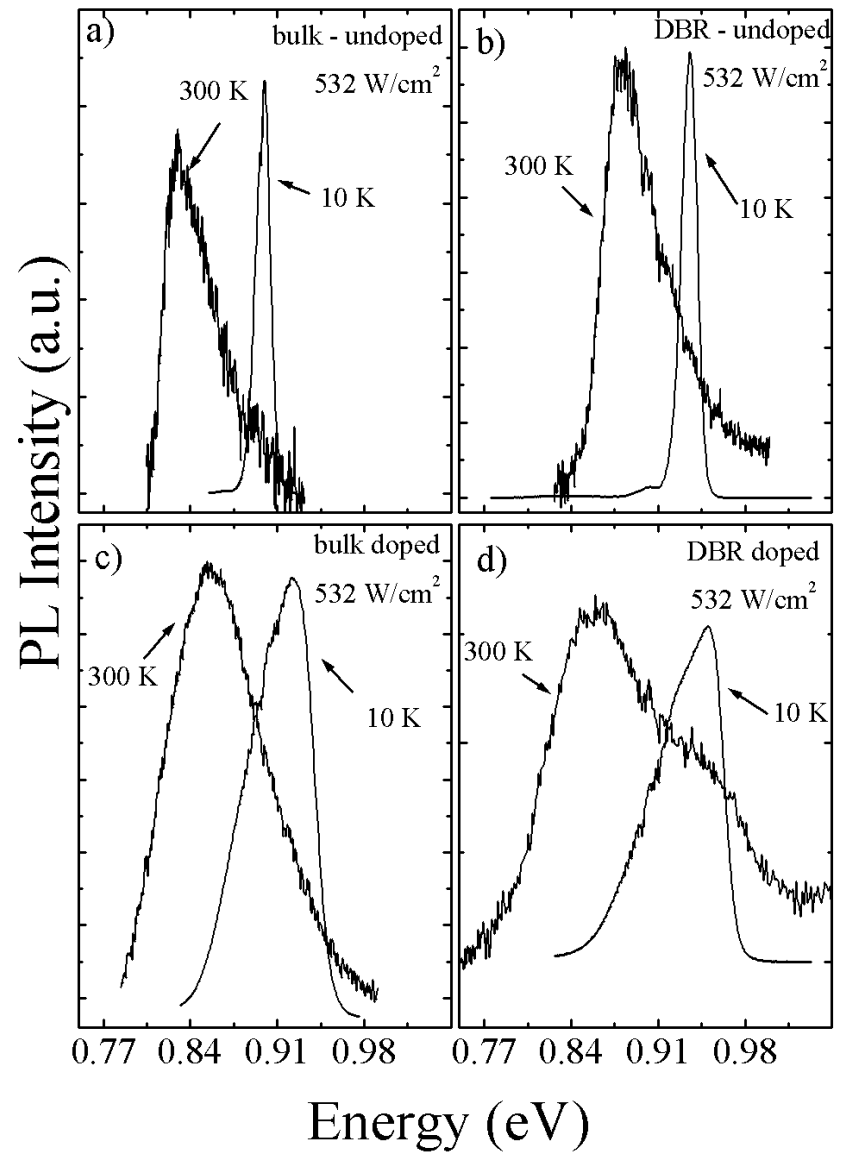

FIG. 3: Photoluminescence curve obtained with laser intensity at 532 $\mathrm{W} / \mathrm{cm}^{2}$ and temperature between $10 \mathrm{~K}$ and $300 \mathrm{~K}$, for samples: a) AlGaAsSb undoped bulk (76N46); b) undoped Bragg mirror (17Q29); c) doped bulk AlGaAsSb (76N39); c) homogeneous doped Bragg mirror (17Q44).

excitation intensity of $532 \mathrm{~W} / \mathrm{cm}^{2}$ as a function of the temperature are shown in Fig. 5c) in a linear scale and in Fig. 5d) in a $\log$ scale. The set of PL spectra in the linear scale shows an explicit "S" behavior for the dominant transition with increasing temperature similar to that observed for undoped and Te doped bulk samples of antimony family epilayers analyzed elsewhere [21]. The PL spectra presented indicate two transitions, one with lower energy peak transition more intense at higher temperatures.

The temperature dependence of the PL dominant peak energies of undoped (76N46), Te doped (76N39) bulk samples and undoped (17Q29) and Te doped (17Q44) Bragg mirrors in the $10 \mathrm{~K}-300 \mathrm{~K}$ range for intensity excitation of $532 \mathrm{~W} / \mathrm{cm}^{2}$ are shown in Fig. 6a). The temperature dependence of dominant transitions in undoped samples is very similar, showing a weak blue shift (1-2 meV) at low temperatures $(\mathrm{T}<100 \mathrm{~K})$. The behavior of the PL peak energies of Te doped bulk alloy as a function of the temperature shows a weak "S" pattern with the first red shift of $\cong 4 \mathrm{meV}$ until $\mathrm{T} \cong 50 \mathrm{~K}$. The peak energy of $n$-type AlGaAsSb layers in the Te doped Bragg mirror shows a stronger " $S$ " behavior with the first red shift of $\cong 13$ $\mathrm{meV}$ to $\mathrm{T} \cong 50 \mathrm{~K}$. The "red/blue/red" behavior is characteristic of materials with electrostatic fluctuations and is stronger as the amplitude of the potential fluctuation grows. This effect can be observed in undoped GaAsSb and AlGaAsSb samples [9]. The presence of $\mathrm{Al}$ in the quaternary alloy increases the fluctuation of the electrostatic fluctuation, increasing the variation of recombination energies as a function of temperature thus decreasing intensity excitations at low temperatures and reinforcing the "S" behavior. Here the presence of the unexpected macro fluctuation in the incorporated Te observed by SIMS in the doped Bragg mirror can explain the stronger "S" behavior in the temperature dependence of PL transitions.

Figure 6b) shows the temperature dependence of the FWHM (Full Width Half Maximum) in all samples. The doped samples shows a stronger broadening ( $\cong 40 \mathrm{meV}$ ) as compared to that observed for undoped samples. The FWHM of the emission spectra of all samples shows an increasing broadening in the $10 \mathrm{~K}-300 \mathrm{~K}$ temperature interval. The stronger broadening observed for undoped Bragg mirror as compared to the undoped AlGaAsSb layer can be associated to the higher $\mathrm{Al}$ concentration in the quaternary layer of the mirror. The behavior of FWHM as a function of the temperature, in the two doped samples present some differences. The FWHM of the doped layer is very similar to that of the undoped with increasing temperature. The FWHM of the Bragg mirror shows a gradual increasing with increasing temperature, reaching a plateau in the temperature range between $50 \mathrm{~K}$ and $100 \mathrm{~K}$ and increasing again after the plateau. The plateau observed indicates two competing recombination channels in the respective temperature range. One channel is dominant at the low temperature limit and the other is dominant at the high temperature limit. This is a characteristic behavior of the competition between donor-acceptor-pair or a quasi-donoracceptor-pair transitions and free to bound transitions with increasing temperatures discussed more systematically elsewhere for the antimony family [9].

Reflectivity measurements for both undoped and doped Bragg mirrors are shown in Fig. 7. Results show that even for a small number of periods it is possible to obtain a relatively high reflectivity and a wide stop band for the Al$\mathrm{GaAsSb} / \mathrm{AlAsSb}$ system. This is associated with the high index contrast $(\cong 0.54)$ between the quaternary and ternary alloys. The highest reflectivity (widest stop band) was obtained for the undoped Bragg mirror, however the reflectivity difference is not very large between the two mirrors changing from $0.91(0.39 \mu \mathrm{m})$ for the undoped mirror to $0.90(0.37 \mu \mathrm{m})$ for the doped mirror. The decrease in reflectivity in the doped sample is associated with a free carrier absorption due to the presence of Te.

The current versus voltage characteristic, perpendicular to the interface, in the doped Bragg mirror is presented in Fig. 8. At a current density of $1 \mathrm{KA} / \mathrm{cm}^{2}$, a typical threshold value for an edge-emitting laser, a voltage drop per period of $138 \mathrm{mV}$ is obtained. The current flow is limited by the potential barriers formed at the interface between quaternary and ternary alloys determined principally by the conduction band off set $(\sim 0.470$ $\mathrm{eV}$ ) and the doping level of the material system. Then the transport properties are dominated by the $\Gamma-\mathrm{X}$ barrier height. The macro fluctuation observed by SIMS introduces a dis- 


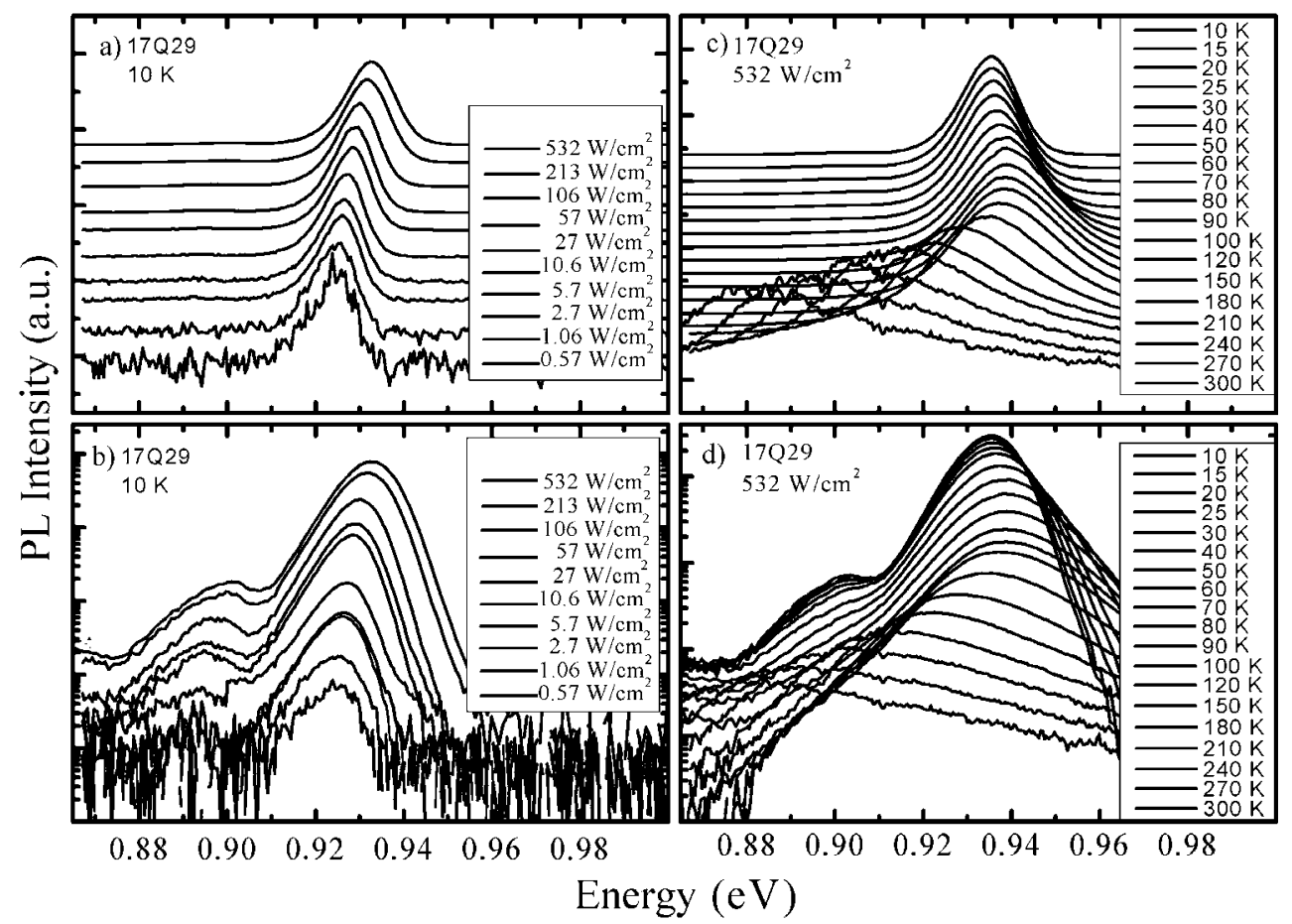

FIG. 4: Photoluminescence curve with laser intensity and temperature dependences, for undoped Bragg mirror sample (17Q29).

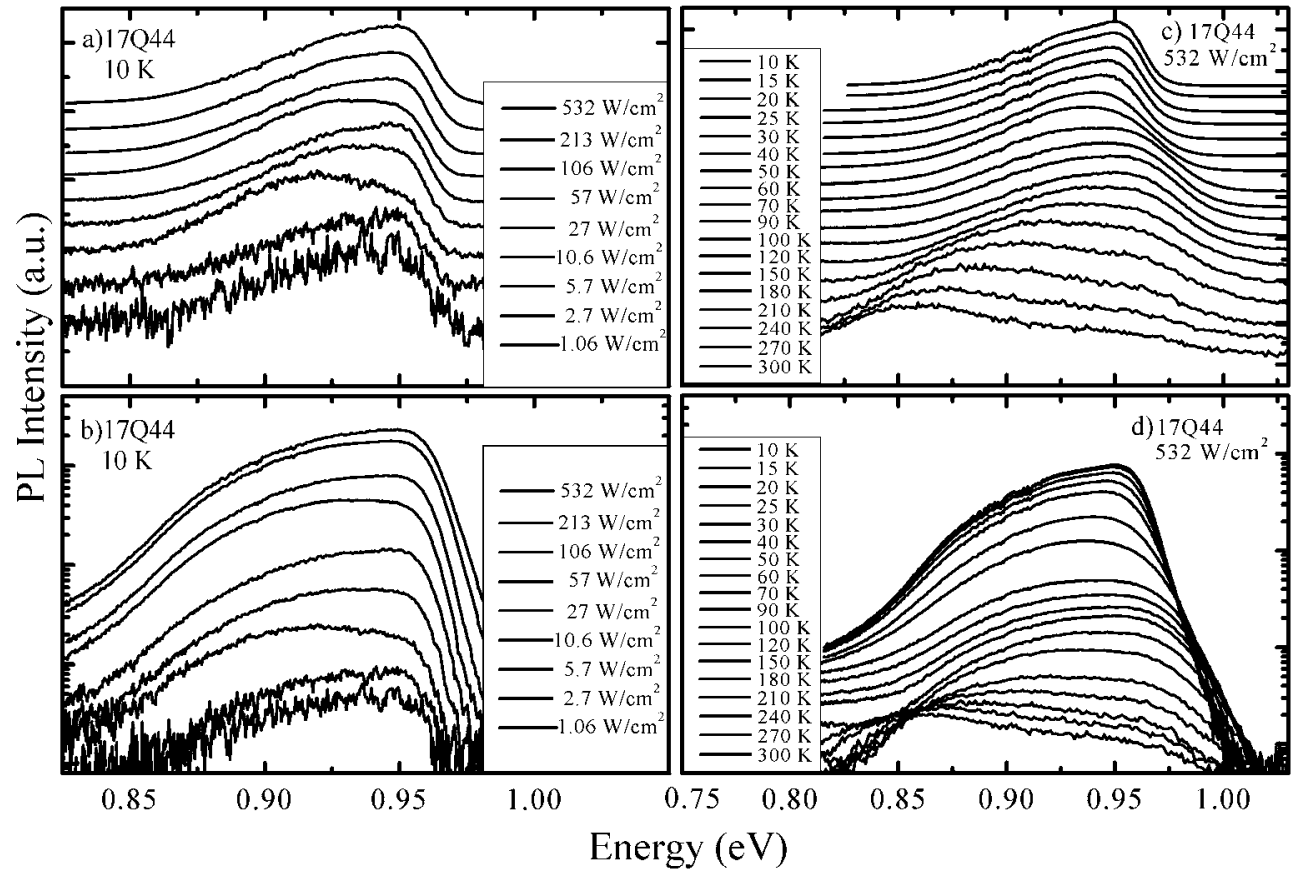

FIG. 5: Photoluminescence curve with laser intensity and temperature dependences, for doped Bragg mirror sample (17Q44).

turbance in the conduction and valence band edges causing strong electrostatic fluctuations and/or scattering centers that can also contribute to an increase in serial resistance. The noncompletely ohmic character of the IxV curve can be associated with several factors such as the asymmetric incorporation of Te, higher at the end of the quaternary layer due to macrofluc- tuations of the dopant detected by SIMS and, principally, by the high off set of the conduction band in the InP/AlGaAsSb interface, as seen in the Fig. 1. The injection of carriers can be improved by using modulation of the doping profile or gradual compositional variation of the components in the Bragg mirror at the heterointerfaces. The growth conditions can be 


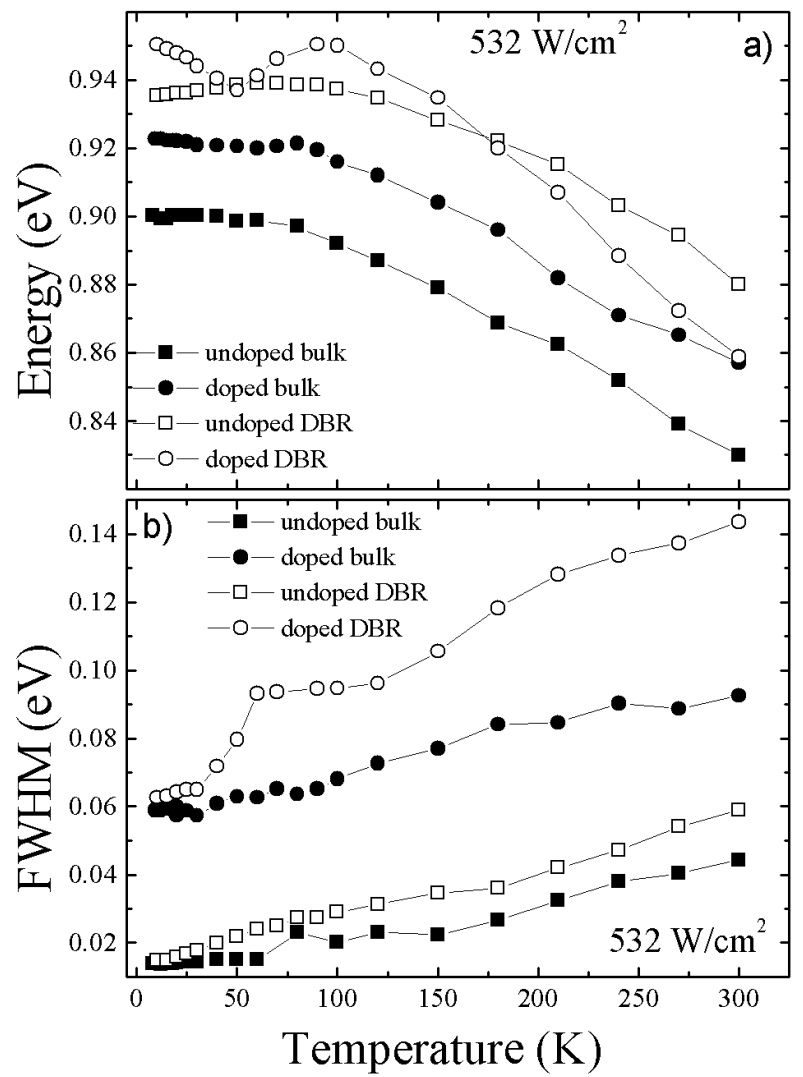

FIG. 6: Energy and FWHM dependences of main peak of PL with the temperature, for undoped bulk AlGaAsSb (76N46), doped bulk AlGaAsSb (76N39), undoped Bragg mirror (17Q29) and doped Bragg mirror (17Q44), obtained with laser intensity at $532 \mathrm{~W} / \mathrm{cm}^{2}$.

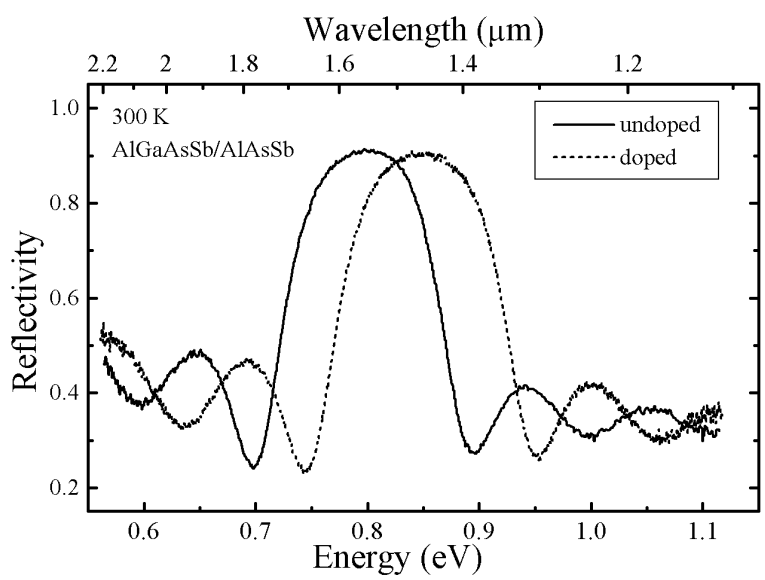

FIG. 7: Reflectivity curves for DBRs samples with 6,5 periods, obtained at $300 \mathrm{~K}$.

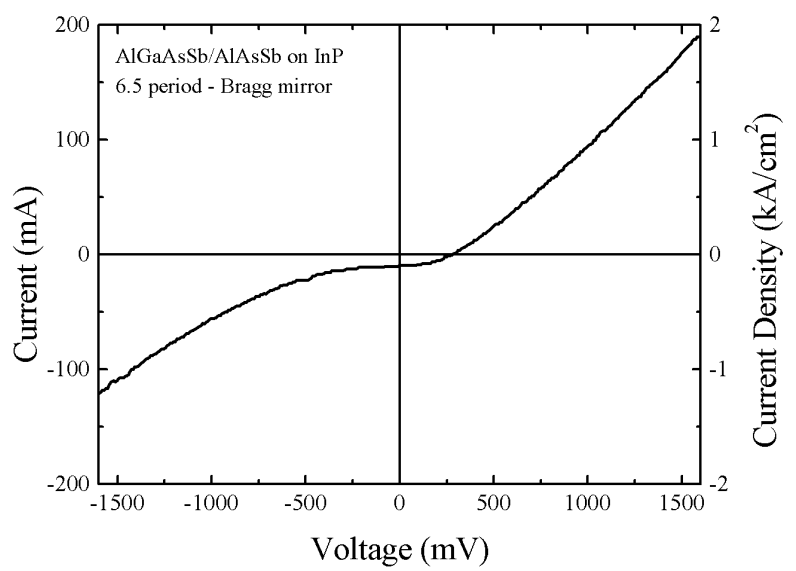

FIG. 8: Current $x$ Voltage curve of the homogeneous doped Bragg mirror (17Q44) at $300 \mathrm{~K}$.

also optimized to improve the reflectivity and conduction parameters.

\section{CONCLUSION}

We have investigated Bragg mirrors of undoped and Te doped AlGaAsSb/AlAsSb system with 6.5 periods and undoped and Te doped bulk samples of AlGaAsSb using SIMS, photoluminescence, reflectivity and IxV measurements. The optical transitions in the PL spectra of AlGaAsSb layers in undoped and Te doped Bragg mirror show similar behavior and lineshape to that found in bulk samples of undoped and Te doped AlGaAsSb. The optical transitions are associated with donor and acceptor recombinations in alloys with potential fluctuations consistent with the quasi-donor-acceptor-pair (QDAP) model. The macrofluctuations in the incorporated Te, associated probably with the growth process, introduce another source of disturbance that intensify the electrostatic potential fluctuation in addition to the characteristic edge band variations given by the alloy and by doping raising the amplitude of the "S" behavior. The presence of macrofluctuations in the dopant incorporations can also generate alternative recombination channels and/or scattering centers harmful to the optical and electrical properties of the Bragg mirrors. Moreover, the asymmetric pattern of the dopant incorporation can facilitate the electron conduction in one direction.

The doping level employed, the standard electrostatic fluctuations generally found in undoped and doped alloys due the presence of a high number de components in the alloys employed, and the dopants and the macrofluctuation observed in the Te incorporated in the Te doped Bragg mirror seem not to affect its reflectivity much. This is associated to the high index contrast $(\cong 0.54)$ between the quaternary and ternary alloys of the AlGaAsSb/AlAsSb system. Hence, the AlGaAs/AlAsSb seems a very attractive option for VCSEL technology. More systematic work is needed to optimize growth conditions and to the improvement of charge carriers injection at the interfaces. 


\section{Acknowledgements}

The authors wish to thank the financial sup- port granted by the Brazilian agencies CAPES, CNPq, Fundação Araucária and FBB.
[1] B. Lambert, Y. Toudic, Y. Rouillard, M. Baudet, B. Guenais, B. Deveaud, I. Valiente, and J. C. Simon, Appl. Phys. Lett. 64, 690 (1994).

[2] O. Blum, I. J. Fritz, L. R. Dawson, and T. J. Drummond, Electron. Lett. 31, 1247 (1995).

[3] N. Kuze, H. Goto, M. Matsui, I. Shibasaki, and H. Sakaki, J. Cryst. Growth 175/176, 868 (1997).

[4] D. O. Toginho Filho, I. F. L. Dias, J.L.Duarte, S. A. Lourenço, L. C. Poças, E. Laureto, J. C. Harmand, Superlattices Microstruct. 31, 277 (2002).

[5] F. Genty, G. Almuneau, L. Chusseau, Wilk, S. Gaillard G. Boissier, P. Grech, and J. Jacquet, J. Cryst. Growth 201/202, 1024 (1999).

[6] I. F. L. Dias, B. Nabet, A. Kohl, and J. C. Harmand, Electron. Lett. 33, 716 (1997).

[7] T. H. Chiu, W. T. Tsang, S. N. G. Chu, J. Shah, and J. A. Ditzenberger, Appl. Phys. Lett. 46, 408 (1985).

[8] B. Shin, A. Lin, K. Lappo, R. S. Goldman, M. C. Hanna, S. Francoeur, A. G. Norman, and A. Mascarenhas, Appl. Phys. Lett. 80, 3292 (2002).

[9] D. O. Toginho Filho, I. F. L. Dias, E. Laureto, J. L. Duarte, S.
A. Lourenço, L. C. Poças, S. S. Prabhu, and J. Klem, J. Appl. Phys. 97, 123702 (2005).

[10] J. B. Krieger, and M Nightingale, Phys. Rev. B 4, 1266 (1971).

[11] N. F. Mott, Proc.Phys. Soc. (London) 62, 419 (1949).

[12] N. F. Mott, Rev. Mod. Phys. 40, 677 (1968).

[13] E. Burstein, Phys. Rev. 93, 632 (1954).

[14] T. S. Moss, Proc.Phys. Soc. (London) B76, 775 (1954).

[15] J. I. Pankove, and P. Aigrain, Phys. Rev. 126, 956 (1962).

[16] R. A. Abram, G.J.Rees, and B.L.H. Wilson, Adv. Phys. 27, 799 (1978).

[17] K. F. Berggren, and B. E. Sernelius, Phys. Rev. B 24, 1971 (1981).

[18] J. C. Harmand, Kohl, M. Juhel, and G. Le Roux, J. Cryst. Growth 175, 372 (1997)

[19] L. Chusseau, G. Almuneau, and F. Genty, Recent Research developments in Quantum Electronics 1, 85 (1999).

[20] P. S. Dutta, H. L. Bhat, and V. Kumar, J. Appl. Phys. 81, 5821 (1997).

[21] D. O. Toginho Filho, I. F. L. Dias, J. L. Duarte, E. Laureto, and Jean C. Harmand, Braz. J. Phys. 35, 999 (2005). 